\title{
Quotient Seminear-Rings
}

\author{
Fawad Hussain', Muhammad Tahir'2, Saleem Abdullah² and Nazia Sadiq² \\ 'Department of Mathematics, Abbottabad University of Science and Technology, Pakistan; \\ fawad.hussain30@gmail.com \\ 2Department of Mathematics, Hazara University Mansehra, Pakistan; m_tahir155@yahoo.com; \\ saleemabdullah81@yahoo.com; nazia.sadiq06@gmail.com
}

\begin{abstract}
The study of a seminear-ring was started in 1967. Seminear-ring is the generalization of semiring and nearing. It is known that in a semiring the quotient structure is constructed by congruence relations through c-ideals and homomorphisms. We apply the said congruence relations to a seminear-ring and get quotient structure of a seminear-ring. The aim of this paper is to discuss quotient seminear-rings in two different ways. We study congruence relations, homomorphisms and ideals. We show that each homomorphism and c-ideal define a congruence relation on seminear-rings. At the end, we discuss quotient seminear-rings.
\end{abstract}

Keywords: Seminear-ring, c-Ideals, Homomorphism, Congruence Relation, Quotient Seminear-Ring.

\section{Introduction}

The study of semirings was started by the German Mathematician Dedekind ${ }^{1}$. They were later studied by different Mathematicians, particularly by the American Mathematician Vandiver. He did work very hard on semirings. He wanted to accept a semiring as fundamental and best algebraic structure ${ }^{2}$. He was not successful because of few reasons and semirings had fallen into disuse. However, during the late 1960's real and significant applications were found of semirings in several fields. These fields include: automata theory, optimization theory, graph theory, the theory of discrete event dynamical system, coding theory, analysis of computer programs, algebras of formal processes and generalized fuzzy computation. The detail of these can be found $\mathrm{in}^{3}$. Later on, different people worked on semirings and explored many interesting properties of semirings. $\operatorname{In}^{4}$ the rough set theory has been applied to semirings while in some applications of a semiring have been studied. In the paper $^{6}$ the authors introduced the notion of a seminearring which was a generalization of a semiring and then explored some properties of seminear-rings. Especially the authors of ${ }^{\underline{6}}$ discussed homomorphisms in seminear- rings and explored some interesting properties. There is another intersecting paper ${ }^{7}$ in which the author discussed seminear-rings as well as semi-near fields. Further different people in $\frac{8-11}{{ }^{8}}$ worked on seminear-rings and explored many interesting and elegant properties. The author of ${ }^{8.9}$ discussed substructures in seminear-rings. The author of ${ }^{11}$ discussed radicals in seminear-rings while the authors of ${ }^{10}$ discussed weekly regular seminear-rings. Before four years ago Perumal and Balakrishnan discussed left bipotent seminear-rings in $\frac{12}{2}$ and discussed left duo seminear-rings in $^{\frac{13}{3}}$ and explored some useful properties. In this paper, we study quotient structure of a seminearring while different quotient algebraic structures have been studied in $\frac{14,15}{}$.

\section{Preliminaries}

This portion contains some of the basic definitions, fundamental results, and reviews some of the background material, which will be used in the coming sections. In 1967, Hoorn and Rootoselaar introduced the notion of a semiring which was a generalization of a semiring. We start with the following definition of a semiring which has been taken from ${ }^{?}$.

*Author for correspondence 


\subsection{Definition}

A seminear-ring is a non-empty set $\boldsymbol{R}$ together with two binary operations ' + ' and '"' such that $(\boldsymbol{R},+)$ and $\left(\boldsymbol{R},,^{\circ}\right)$ are semigroups such that

$$
(a+b) \cdot c=(a \cdot c)+(b \cdot c) \text { holds for all } a, b, c \in \boldsymbol{R} .
$$

It will be more suitable to call what we have just defined a right seminear-ring. In the same way, we may define a left seminear-ring in which one use left distributive law instead of right distributive law. In this paper, we shall keep to right seminear-rings throughout. To understand the notion of semirings, we give some examples.

\subsection{Examples}

(i) All rings and semirings are seminear-rings.

(ii) Let $N$ be the set of natural numbers, then $N$ is a semiring with ordinary addition and multiplication of numbers.

We are now giving a non-trivial example of a seminear-ring.

(iii) Let $N$ be the set of natural numbers. Let

$$
\Omega=\left\{\left[\begin{array}{ll}
a & b \\
\mathrm{c} & d
\end{array}\right]: a, b, c, d \in N\right\}
$$

and define, $A+B=A B$ and $A \cdot B=A$ for all $A, B \in \Omega$. Then $(\Omega,+, \cdot)$ is a seminear-ring.

We are now going to define subseminear-rings and ideals in seminear-rings. Firstly we are going to define subseminear-rings for which we don't have a specific reference.

\subsection{Definition}

A non-empty subset $S$ of a seminear-ring $(R,+, \cdot)$ is called a subseminear-ring of $\boldsymbol{R}$ if $\boldsymbol{S}$ itself is a seminear-ring under the operations of $\boldsymbol{R}$ when restricted to $S$.

It should be noted that very seminear-ring $(\boldsymbol{R},+, \cdot)$ has at least one subseminear-ring, i.e. $\boldsymbol{R}$ itself called the trivial subseminear-ring. All other subseminear-rings are called non-trivial subseminear-rings. We are now going to present some properties of subseminear-rings. The result given below gives us necessary and sufficient conditions for subseminear-rings and for which we don't have a specific reference.

\subsection{Theorem}

Let $(\boldsymbol{R},+, \cdot)$ be a seminear-ring, then a non-empty subset $\boldsymbol{S}$ of $\boldsymbol{R}$ is called a subseminear-ring of $\boldsymbol{R}$ if and only if (i) $x+y \in S$ for all $x, y \in S$,

(ii) $x \cdot y \in S$ for all $x, y \in S$.

\section{Proof}

The proof is easy and is left for the readers.

We are now going to define ideals. The following definition has been taken from the paper ${ }^{\circ}$.

\subsection{Definition}

A non-empty subset $I$ of a seminear-ring $\left(R,+,^{*}\right)$ is called a left (respectively right) ideal of $\boldsymbol{R}$ if the following conditions hold:

(i) $x+y \in I$ for all $x, y \in I$,

(ii) $a \cdot x \in I(x \cdot a \in I)$ for all $x \in I$ and $a \in R$.

$\boldsymbol{I}$ is called a two-sided ideal or simply an ideal of $\boldsymbol{R}$ if it is both left as well as right ideal of $\boldsymbol{R}$.

Note that it is obvious from the above definition that every ideal is a subseminear-ring and we know that every subseminear-ring is a seminear-ring. Thus, it follows that every ideal is a seminear-ring.

We are now going to define c-ideals in seminearrings. The idea of this definition comes from the paper ${ }^{16}$ in which the authors define c-ideals in semirings.

\subsection{Definition}

An ideal $\boldsymbol{I}$ of a seminear-ring $(\boldsymbol{R},+, \cdot)$ is called a c-ideal of $\boldsymbol{R}$ if for any $a, b \in \boldsymbol{R}$, there exist $x, y \in \boldsymbol{I}$ such that $a+b+x=y+b+a$.

Like homomorphisms of other algebraic structures such as semirings and rings, homomorphisms of seminear-rings are those maps which preserve the binary operations. We now give a proper definition of a seminear-ring homomorphism. The following definition is taken from ${ }^{8}$.

\subsection{Definition}

Let $(\boldsymbol{R},+, \cdot)$ and $(\boldsymbol{S}, \Theta, \odot)$ be two seminear-rings. A mapping $\mu: R \rightarrow S$ from $R$ to $S$ is called a homomorphism if and only if:

(i) $\mu(a+b)=\mu(a) \oplus \mu(b)$

(ii) $\mu(a \cdot b)=\mu(a) \odot \mu(b)$

for all $a, b \in \boldsymbol{R}$.

It should be noted that if $\boldsymbol{R}=\boldsymbol{S}$, then the homomorphism $\mu$ is known as an endomorphism. If $\mu$ is homomorphism as well as onto, then $\mu$ is called an epimorphism. If $\boldsymbol{\mu}$ is homomorphism as well as one-one, then $\mu$ is called a monomorphism. A homomorphism $\mu$ is called an isomorphism if it is epimorphism as well as 
monomorphism. An isomorphism $\mu$ is called an automorphism if $\boldsymbol{R}=\boldsymbol{S}$. If $\mu: \boldsymbol{R} \rightarrow \boldsymbol{S}$ is an isomorphism then we say that $R$ is isomorphic to $S$ and write:

$$
R \cong S
$$

\section{Congruence Relations}

This section concerned with the congruence relations on seminear-rings. We define congruence relations and show that each homomorphism defines a congruence relation on seminear-rings. The idea of this section comes from the book ${ }^{17}$ in which the author does similar calculations for semigroups.

\subsection{Definition}

Let $(\boldsymbol{R},+, \cdot)$ be a seminear-ring. A relation $\rho$ on $\boldsymbol{R}$ is called left compatible if for all $s, t, a \in R$ such that $(s, t) \in \rho$ implies that $(a+s, a+t),(a \cdot s, a \cdot t) \in \rho$.

$\rho$ is called right compatible if for all $s, t, b \in R$ such that $(s, t) \in \rho$ implies that $(s+b, t+b),(s \cdot b, t \cdot b) \in \rho$.

$\rho$ is called compatible if for all $s, t, u, v \in R$ such that (s, $t),(u, v) \in$ pimplies that $(s+u, t+v),(s \cdot u, t \cdot v) \in \rho$.

A left (respectively right) compatible equivalence relation is said to be a left (respectively right) congruence relation. A compatible equivalence relation is said to a congruence relation.

In order to understand the above concept, we give an example.

\subsection{Example}

Consider the seminear-ring $(\Omega,+, \cdot)$ of Example 2.2 (iii). Let $\rho=\{(\boldsymbol{A}, \boldsymbol{B}): \boldsymbol{A}=\boldsymbol{B}\}$ be a relation on $\Omega$. Then one can show that $\rho$ is a congruence relation on $\Omega$.

We are now going to state and prove a result which gives equivalent conditions for congruence relations.

\subsection{Proposition}

A relation $\rho$ on a seminear-ring $R$ is a congruence relation if and only if it is both left as well as aright congruence relation.

\section{Proof}

Assume that $\rho$ is a congruence relation on $R$. Choose $s, t$, $a \in \boldsymbol{R}$ such that $(s, t) \in \rho$, then $(a+s, a+t),(a \cdot s, a \cdot t) \in$ $\rho$, because $(a, a) \in \rho$. It follows that $\rho$ is a left congruence relation.
Similarly one can show that $\rho$ is a right congruence relation.

Conversely, suppose that $\rho$ is both left as well as right congruence relation. Choose $s, t, u, v \in \boldsymbol{R}$ such that $(s, t)$, $(u, v) \in \rho$. This implies that $(s+u, t+u),(s \cdot u, t \cdot u) \in \rho$, because $\rho$ is right compatible and $(t+u, t+v),(t \cdot u, t \cdot v) \in \rho$, because $\rho$ is left compatible. It follows that $(s+u, t+v)$, $(s \cdot u, t \cdot v) \in \rho$, because $\rho$ is transitive. This completes the proof.

\section{Congruence Relations and Homomorphisms}

In this section we state and prove a result which says that corresponding to every homomorphism there is a congruence relation. The result is important because once we get it we can get quotient seminear-rings. At the end we prove analogues of the isomorphism theorems.

\subsection{Theorem}

If $h$ is a homomorphism from a seminear-ring $(\boldsymbol{R},+, \cdot)$ to a seminear-ring $(S, \oplus, \odot)$, then $h$ defines a congruence relation $\rho$ on $\boldsymbol{R}$ given by $(r, s) \in \rho$ if and only if $h(r)=h(s)$.

\section{Proof}

First we show that $\rho$ is an equivalence relation. As $h(r)=$ $h(r)$ for all $r \in \boldsymbol{R}$, therefore $(r, r) \in \rho$ and so the relation $\rho$ is reflexive. If $(r, s) \in \rho$ for some $r, s \in \boldsymbol{R}$, then $h(r)=h(s)$ and this implies that $h(s)=h(r)$. Thus, $(s, r) \in \rho$ and so the relation $\rho$ is symmetric. Now if $(r, s),(s, t) \in \rho$, then according to the definition $h(r)=h(s)$ and $h(s)=h(t)$ and this gives us $h(r)=h(t)$. This implies that $(r, t) \in \rho$ and so the relation $\rho$ is transitive. Now let $(r, s),(t, u) \in \rho$, then according to the definition $h(r)=h(s)$ and $h(t)=h(u)$. Now $h(r+t)=h(r) \oplus h(t)=h(s) \oplus h(u)=h(s+u)$. It follows that $(r+t, s+u) \in \rho$. In the same way one can show that $(r \cdot t, s \cdot u) \in \rho$. Thus, the relation $\rho$ is compatible. This completes the proof.

Now let $\rho$ be an equivalence relation on a set $\boldsymbol{X}$. Then the equivalence class corresponding to the element $x$ of $\boldsymbol{X}$ is represented by the symbol $x \rho$ and is defined as follows:

$$
x \rho=\{y \in X(x, y) \in \rho\} .
$$

If $\rho$ is a congruence relation on a seminear-ring $R$, then we say that $x \rho$ is called a congruence class corresponding 
to the element $x$ of $\boldsymbol{R}$. Let $\boldsymbol{R} / \mathrm{\rho}$ be the set of all congruence classes. That is

$$
\boldsymbol{R} / \rho=\{x \rho: x \in \boldsymbol{R}\} .
$$

We are now going to state a result which is taken from the book $^{18}$ and will be used later. The result is true for classes but as we know that every class is a set, so in particular, it is true for sets as well.

\subsection{Lemma}

Let $\boldsymbol{X}$ be a set and $\rho$ an equivalence relation on $\boldsymbol{X}$. Then $x \rho$ $=y \rho$ if and only if $(x, y) \in \rho$.

Let $x, y \in \boldsymbol{R}$ and $x \rho, y \rho$ be the congruence classes corresponding to the elements $x$ and $y$, then we can define binary operations on the quotient set $\boldsymbol{R} /$ pin the following way:

$$
x \rho+y \rho=(x+y) \rho \text { and } x \rho \cdot y \rho=(x \cdot y) \rho .
$$

These operations are well defined, since for all $w, x, y, z \in$ $\boldsymbol{R}$ such that $w \rho=y \rho$ and $x \rho=z \rho$, then by Lemma 4.2, ( $w$, $y) \in \rho$ and $(x, z) \in \rho$. This implies that $(w+x, y+z),(w$. $x, y \cdot z) \in \rho$, since $\rho$ is a congruence relation. Thus, again Lemma 4.2, this implies that $(w+x) \rho=(y+z) \rho$ and $(w$. $x) \rho=(y \cdot z) \rho$. Further, we have

\section{Associative laws}

Choose $x, y, z \in R$ such that $x \rho, y \rho, z \rho \in R / \rho$, then

(i) With respect to ' + ':

$$
\begin{aligned}
(x \rho+y \rho)+z \rho & =(x+y) \rho+y \rho \\
& =((x+y)+z)) \rho \\
& =((x+(y+z)) \rho \\
& =x \rho+(y+z) \rho \\
& =x \rho+(y \rho+z \rho) .
\end{aligned}
$$

(ii) With respect to ' $?$ ':

$$
\begin{aligned}
(x \rho \cdot y \rho) \cdot y \rho & =(x \cdot y) \rho \cdot z \rho \\
& =((x \cdot y) \cdot z)) \rho \\
& =((x \cdot(y \cdot z)) \rho \\
& =x \rho \cdot(y \cdot z) \rho \\
& =x \rho \cdot(y \rho \cdot z \rho) .
\end{aligned}
$$

Thus, $(\boldsymbol{R} / \rho,+)$ and $\left(\boldsymbol{R} / \rho,^{*}\right)$ are: semigroups.

\section{Right distributive law}

Choose $x, y, z \in R$ such that $x \rho, y \rho, z \rho \in R / \rho$, then

$$
\begin{aligned}
(y \rho+z \rho) \cdot x \rho & =(y+z) \rho \cdot x \rho \\
& =((y+z) \cdot x) \rho \\
& =(y \cdot x+z \cdot x) \rho \\
& =(y \cdot x) \rho+(z \cdot x) \rho
\end{aligned}
$$

$$
=y \rho \cdot x \rho+z \rho \cdot x \rho
$$

Thus $(R / \rho,+, \cdot)$ is a seminear-ring which is called a quotient seminear-ring.

We are now going to state and prove the seminearring analogues of the first, second and third isomorphism theorems. The semigroup equivalents may be found in the book ${ }^{17}$.

\subsection{Theorem [First Isomorphism Theorem]}

Assume that $\rho$ is a congruence relation on a seminearring $\boldsymbol{R}$. Then $R / \rho$ is a seminear-ring under the following binary operations:

$$
x \rho+y \rho=(x+y) \rho \text { and } x \rho \cdot y \rho=(x \cdot y) \rho
$$

for all $x \rho, y \rho \in R / \rho$. The mapping $\rho^{\#}: R \rightarrow R / \rho$ defined by $\rho(x)=x \rho$ for all $x \in \boldsymbol{R}$ is an epimorphism. Let $(\boldsymbol{R},+, \cdot)$ and $(S, \oplus, \odot)$ be seminear-rings and $\Phi: R \rightarrow S$ a homomorphism. Then the relation $\operatorname{ker} \Phi=\{(x, y) \in \boldsymbol{R} \times \boldsymbol{R}: \Phi(x)=\Phi(y)\}$ is a congruence relation on the seminear-ring $R$ and there is a monomorphism $\alpha: R / \operatorname{ker} \Phi \rightarrow S$ such that $\operatorname{ran} \alpha=\operatorname{ran} \Phi$ and the diagram given below commutes.

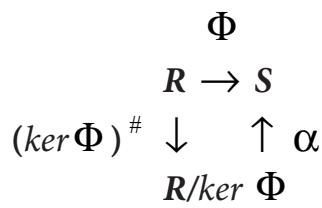

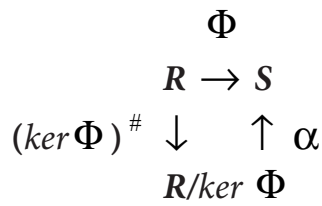

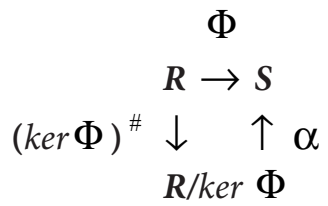

\section{Proof}

We have already proved that $R / \rho$ is a seminear-ring. Now choose $x, y \in R$, then

$\rho^{\#}(x+y)=(x+y) \rho=x \rho+y \rho=\rho^{\#}(x)+\rho^{\#}(y)$

and

$\rho^{\#}(x \cdot y)=(x \cdot y) \rho=x \rho \cdot y \rho=\rho^{\#}(x) \cdot \rho^{\#}(y)$.

It follows that $\rho^{\#}$ is a homomorphism. $\operatorname{ker} \Phi$ is a congruence relation on $\boldsymbol{R}$ by Theorem 4.1. Now define $\alpha: R / \operatorname{ker} \Phi \rightarrow S$ by $\alpha(x k e r \Phi)=\Phi(x)$. Then $\alpha$ is well defined as well as one-one, as for all $x k e r \Phi$, $y k \operatorname{ker} \Phi \in \boldsymbol{R} / \operatorname{ker} \Phi$

$$
\begin{aligned}
& x \operatorname{xkr} \Phi=y \operatorname{ker} \Phi \Leftrightarrow(x, y) \in \operatorname{ker} \Phi \Leftrightarrow \Phi(x)=\Phi(y) \\
& \Leftrightarrow \alpha(x k \operatorname{ler} \Phi)=\alpha(y k \operatorname{ker} \Phi) .
\end{aligned}
$$

$\alpha_{\text {is a homomorphism, as for all } x k e r} \Phi, y k e r \Phi \in R / k e r \Phi$

$$
\begin{aligned}
\left.\left.\alpha_{[(x k e r} \Phi\right)+(y k \operatorname{ker} \Phi)\right] & \left.=\alpha_{[(x+y) k e r} \Phi\right] \\
& =\Phi(x+y) \\
& =\Phi(x) \oplus \Phi(y) \\
& \left.\left.=\alpha_{(x k e r} \Phi\right) \oplus \alpha_{(y k e r} \Phi\right)
\end{aligned}
$$

and 


$$
\begin{aligned}
\alpha[(x \operatorname{ker} \Phi) \cdot(y \operatorname{ker} \Phi)] & =\alpha[(x \cdot y) \operatorname{ker} \Phi] \\
& =\Phi(x \cdot y) \\
& =\Phi(x) \odot \Phi_{(y)} \\
& =\alpha_{(x k \operatorname{ker} \Phi)} \odot \alpha_{(y \operatorname{ker} \Phi)}
\end{aligned}
$$

Obviously $\operatorname{ran} \alpha=\operatorname{ran} \Phi$ and from the definition it is clear that for all $x \in \boldsymbol{R}$

$$
\alpha(\operatorname{ker} \Phi))^{\#}(x)=\alpha(x \operatorname{ker} \Phi)=\Phi(x) .
$$

That is, the diagram commutes.

\subsection{Theorem (Second Isomorphism Theorem)}

Let $(\boldsymbol{R},+, \cdot)$ and $(\boldsymbol{S}, \Theta, \odot)$ be seminear-rings and $\rho$ a congruence relation on the seminear-ring $\boldsymbol{R}$. Assume that $\Phi: R \rightarrow S$ is a homomorphism such that $\rho \subseteq$ ker $\Phi$ . Then there is a unique homomorphism $\beta: R / \rho \rightarrow S$ such that $\operatorname{ran} \beta=\operatorname{ran} \Phi$ and the diagram given below commutes.

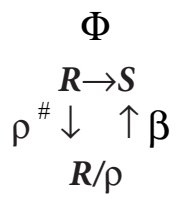

\section{Proof}

Define the mapping $\beta: R / \rho \rightarrow S$ by $\beta(x \rho)=\Phi(x)$ for all $x \rho \in R / \rho$. Then $\beta$ is well-defined, because for all $x \rho, y \rho \in$ $R / \rho, x \rho=y \rho \Rightarrow(x, y) \in \rho \subseteq \operatorname{ker} \Phi \Rightarrow \Phi(x)=\Phi(y) \Rightarrow$ $\beta(x \rho)=\beta(y \rho)$.

$\beta$ is a homomorphism, since for $x \rho, y \rho \in R / \rho$,

$\beta(x \rho+y \rho)=\beta[(x+y) \rho]=\Phi(x+y)=\Phi(x) \oplus \Phi(y)$ $=\beta(x \rho) \oplus \beta(y \rho)$

and

$\beta(x \rho \cdot x \rho)=\beta[(x \cdot y) \rho]=\Phi(x \cdot y)=\Phi(x) \odot \Phi(y)$ $=\beta(x \rho) \odot \beta(y \rho)$.

Now $\beta \rho^{\#}(x)=\beta(x \rho)=\Phi(x)$. It follows that the diagram commutes. Clearly $\operatorname{ran} \beta=\operatorname{ran} \Phi$. To show the uniqueness, let $\alpha: R / \rho \rightarrow S$ be another homomorphism such that $a \rho^{\#}=\Phi$. Let $x \in R$, then

$$
a \rho^{\#}(x)=\Phi(x)=\beta \rho^{\#}(x) \Rightarrow \alpha(x \rho)=\beta(x \rho) \Rightarrow \alpha=\beta \text {. }
$$

\subsection{Theorem [Third Isomorphism Theorem]}

Let $\boldsymbol{R}$ be a seminear-ring. Assume that $\rho$ and $\sigma$ are congruence relations on $\boldsymbol{R}$ such that $\rho \subseteq \sigma$. Then

$$
\sigma / \rho=\{(x \rho, y \rho) \in \boldsymbol{R} / \rho \times \boldsymbol{R} / \rho:(x, y) \in \sigma\}
$$

is a congruence relation on $R / \rho$ and

$$
R / \rho \mid \sigma / \rho \cong R / \sigma \text {. }
$$

\section{Proof}

First, we show that $\sigma / \rho$ is a congruence relation. Choose $r \in \boldsymbol{R}$, then $(r, r) \in \sigma$, since $\sigma$ is reflexive. This implies that $(r \rho, r \rho) \in \sigma / \rho$, so $\sigma / \rho$ is reflexive. Now choose $r, s \in \boldsymbol{R}$ such that $(r \rho, s \rho) \in \sigma / \rho$, then $(r, s) \in \sigma$ and this implies $(s, r) \in \sigma$, since $\sigma$ is symmetric. It follows that $(s \rho, r \rho) \in \sigma / \rho$, so $\sigma / \rho$ is symmetric. Now choose $r, s, t \in \boldsymbol{R}$ such that $(r \rho, s \rho),(s \rho, t \rho) \in \sigma / \rho$, then by definition $(r, s),(s, t) \in \sigma$ and this implies $(r, t) \in \sigma$, as $\sigma$ is transitive. It follows that $(r \rho, t \rho) \in \sigma / \rho$, so $\sigma / \rho$ is transitive. Thus, $\sigma / \rho$ is an equivalence relation.

Now choose $r, s, t, u \in \boldsymbol{R}$ such that ( $r \rho, s \rho)$, $(t \rho, u \rho) \in \sigma / \rho$, then by definition $(r, s),(t, u) \in \sigma$. It follows that $(r+t, s+u),(r \cdot t, s \cdot u) \in \sigma$, since $\sigma$ is compatible, and this implies that

$$
((r+t) \rho,(s+u) \rho),((r \cdot t) \rho,(s \cdot u) \rho) \in \sigma / \rho .
$$

It follows that $\sigma /$ pis compatible.

Now define a mapping $\beta: R / \rho \rightarrow R / \sigma$ by $\beta(x \rho)=x \sigma$. Choose $x \rho, y \rho \in R / \rho$, then

$\beta(x \rho+y \rho)=\beta((x+y) \rho)=(x+y) \sigma=x \sigma+y \sigma=\beta(x \rho)+\beta(x \rho)$ and

$\beta(x \rho \cdot y \rho)=\beta((x \cdot y) \rho)=(x \cdot y) \sigma=x \sigma \cdot y \sigma=\beta(x \rho) \cdot \beta(y \rho)$.

It follows that $\beta$ is a homomorphism. So by Theorem 4.3 , there is a monomorphism

$$
\alpha: R / \rho \mid \operatorname{ker} \beta \rightarrow R / \sigma
$$

defined by $\alpha((x \rho) \operatorname{ker} \beta)=x \sigma$. Obviously it is onto. Thus $R / \rho \mid k e r \beta \cong R / \sigma$. Now

$\operatorname{ker} \beta=\{(x \rho, y \rho) \in R / \rho \times R / \rho: \beta(x \rho)=\beta(y \rho)\}$

$=\{(x \rho, y \rho) \in R / \rho \times R / \rho: x \sigma=y \sigma\}$

$=\{(x \rho, y \rho) \in R / \rho \times R / \rho:(x, y) \in \sigma\}$

$=\sigma / \rho$.

This completes the proof.

\section{Congruence Relations and c-Ideals}

In this section, we show that each c-ideal defines a congruence relation on seminear-rings and the idea comes from the paper ${ }^{16}$ in which the author does similar calculations for semirings. 


\subsection{Theorem}

Let $\boldsymbol{I}$ be a c-ideal of a seminear-ring $(\boldsymbol{R},+, \cdot)$. Then, $\boldsymbol{I}$ define a congruence relation $\rho$ on $\boldsymbol{R}$ given by $(r, s) \boldsymbol{\in} \rho$ if and only if there exist $x, y \in I$ such that $r+x=y+s$.

\section{Proof}

Let $r \in \boldsymbol{R}$ and $x \in \boldsymbol{I}$. As $\boldsymbol{I}$ is a c-ideals of $\boldsymbol{R}$, there exists $x_{1}$, $x_{2} \in I$ such that $r+x+x_{1}=x_{2}+x+r$. This implies that $(r, r) \in \boldsymbol{\in}$, since $\boldsymbol{I}$ is an ideal. Let $(r, s) \in \boldsymbol{\rho}$ for some $r, s$ $\boldsymbol{E} \boldsymbol{R}$. Then there are $x_{1}, x_{2} \in \boldsymbol{I}$ such that $r+x_{1}=x_{2}+s$. As $\boldsymbol{I}$ is a c-ideal of $\boldsymbol{R}$, there exist $x_{3}, x_{4}, x_{5}, x_{6} \in I$ such that $r+$ $x_{1}+x_{3}=x_{4}+x_{1}+r$ and $s+x_{2}+x_{5}=x_{6}+x_{2}+s$. This implies $x_{6}+x_{4}+x_{1}+r=s+x_{2}+x_{5}+x_{3}$ which implies that $(s, r) \in$ $\rho$. Let $r, s, t \in \boldsymbol{R}$ such that $(r, s) \in \rho$ and $(s, t) \in \rho$. Then there exist $x_{1}, x_{2}, y_{1}, y_{2} \in I$ such that $r+x_{1}=x_{2}+s$ and $s+y_{1}=y_{2}+t$. This implies that $r+x_{1}+y_{1}=x_{2}+s+y_{1}=$ $x_{2}+y_{2}+t$ which implies $(r, t) \in \rho$. It follows that $\rho$ is an equivalence relation.

Again as $\boldsymbol{I}$ is an ideal of $\boldsymbol{R},(r, s) \boldsymbol{\in} \rho$ for some $r, s \in$ $\boldsymbol{R}$ implies $(t \cdot r, t \cdot s) \in \rho$, and $(r \cdot t, s \cdot t) \in \rho$, for all $t \in \boldsymbol{R}$. Finally let $r, s, t \in \boldsymbol{R}$ such that $(r, s) \in \boldsymbol{\in}$. This implies that $r$ $+x_{1}=x_{2}+s$. for some $x_{1}, x_{2} \in I$. Therefore $t+\left(r+x_{1}\right)+x_{3}$ $=t+\left(x_{2}+s\right)+x_{3}=x_{4}+\left(x_{2}+s\right)+t$ for some $x_{3}, x_{4} \in I$, since $\boldsymbol{I}$ is a c-ideal of $\boldsymbol{R}$. Thus $(t+r, t+s) \boldsymbol{\in} \rho$. Now using $(t+r$, $t+s) \in \rho$ and by using the fact that $\boldsymbol{I}$ is a c-ideal and $\rho$ is symmetric we may get $(r+t, s+t) \in \rho$. Therefore $\rho$ is left as well as right congruence relation. This completes the proof.

Now let $\rho$ be the above congruence relation on the seminear-ring $\boldsymbol{R}$. Assume that $a+\boldsymbol{I}$ represents the congruence class corresponding to the element $a \in R$ and $R / I$ represents the set of all congruence classes, i.e.

$$
\boldsymbol{R} / \mathbf{I}=\{a+I: a \in R\} .
$$

The following result shows that $R / I$ becomes a seminear-ring.

\subsection{Theorem}

Let $(\boldsymbol{R},+, \cdot)$ be a seminear-ring and $\boldsymbol{I}$ c-ideal of $\boldsymbol{R}$. Then $\boldsymbol{R} / \boldsymbol{I}$ is seminear-ring under the following binary operations:

$(a+\boldsymbol{I})+(b+\boldsymbol{I})=(a+b)+\boldsymbol{I}$ and $(a+\boldsymbol{I}) \cdot(b+\boldsymbol{I})=(a \cdot b)+\boldsymbol{I}$.

\section{Proof}

First we show that the above binary operations are welldefined. Choose $a, b, c, d \in \boldsymbol{R}$ such that $a+\boldsymbol{I}=c+\boldsymbol{I}$ and $b+\boldsymbol{I}=d+\boldsymbol{I}$, then Lemma $4.2,(a, c) \in \rho$ and $(b, d) \in \rho$. Thus $(a+b, c+d),(a \cdot b, c \cdot d) \in \rho$, since $\rho$ is a congruence relation. Thus again by Lemma 4.2, this implies that $(a+b)+\boldsymbol{I}=(c+d)+\boldsymbol{I}$ and $(a \cdot b)+\boldsymbol{I}=(c \cdot d)+\boldsymbol{I}$. Further we prove

\section{Associative laws}

Choose $a, b, c \in R$ such that $a+I, b+I, c+I \in R / I$, then

(i) With respect to ' + ':

$$
\begin{aligned}
(a+\boldsymbol{I}+b+\boldsymbol{I})+c+\boldsymbol{I} & =(a+b)+\boldsymbol{I}+c+\boldsymbol{I} \\
& =((a+b)+c))+\boldsymbol{I} \\
& =((a+(b+c))+\boldsymbol{I} \\
& =a+\boldsymbol{I}+(b+c)+\boldsymbol{I} \\
& =a+\boldsymbol{I}+(b+\boldsymbol{I}+c+\boldsymbol{I}) .
\end{aligned}
$$

(ii) With respect to "' ':

$$
\begin{aligned}
(a+\boldsymbol{I} \cdot b+\boldsymbol{I}) \cdot c+\boldsymbol{I} & =(a \cdot b)+\boldsymbol{I} \cdot \boldsymbol{c}+\boldsymbol{I} \\
& =((a \cdot b) \cdot c))+\boldsymbol{I} \\
& =((a \cdot(b \cdot c))+\boldsymbol{I} \\
& =a+\boldsymbol{I} \cdot(b \cdot c)+\boldsymbol{I} \\
& =a+\boldsymbol{I} \cdot(b+\boldsymbol{I} \cdot c+\boldsymbol{I}) .
\end{aligned}
$$

Thus $(\boldsymbol{R} / \mathbf{I},+)$ and $(\boldsymbol{R} / \mathbf{I}, \cdot)$ are semigroups.

\section{Right distributive law}

Choose $a, b, c \in R$ such that $a+I, b+I, c+I \in R / I$, then

$$
\begin{aligned}
(b+\boldsymbol{I}+c+\boldsymbol{I}) \cdot a+\boldsymbol{I} & =(b+c)+\boldsymbol{I} \cdot a+\boldsymbol{I} \\
& =((b+c) \cdot a)+\boldsymbol{I} \\
& =(b \cdot a+c \cdot a)+\boldsymbol{I} \\
& =(b \cdot a)+\boldsymbol{I}+(c \cdot a)+\boldsymbol{I} \\
& =b+\boldsymbol{I} \cdot a+\boldsymbol{I}+c+\boldsymbol{I} \cdot a+\boldsymbol{I}
\end{aligned}
$$

Thus $(\boldsymbol{R} / \mathbf{I},+, \cdot)$ is a seminear-ring which is called quotient seminear-ring.

\section{Conclusion}

If we assume that $(\boldsymbol{R},+, \cdot)$ and $(\boldsymbol{S}, \oplus, \odot)$ are two seminear-rings with additive identities $0_{R}$ and $0_{S}$, then we say that a map $a: R \rightarrow S$ is a homomorphism if it satisfies the conditions of Definition 2.7 along with the condition $a\left(0_{R}\right)=0_{s}$. In this case, we may define kernel of the homomorphism $a$ in the usual way. Further if we assume that $x \odot 0_{s}=0_{s} \odot x=0_{s}$ for all $x \in S$, then we may prove that kernel is an ideal but we don't know that whether it is a c-ideal or not. Also we know that intersection of two ideals of a seminear-ring $\boldsymbol{T}$ is either empty or an ideal of $\boldsymbol{T}$ but we don't know that whether the intersection of two c-ideals is a c-ideal or not. Therefore we are unable to prove the analogues of the well known isomorphism theorems. 


\section{References}

1. Dedekind R. Uber die Theorie der ganzenalgebraiscen Zahlen, Springer Fachmedien Wiesbaden, 1964, p. 297-13.

2. Vandiver HS. Note on a simple type of algebra in which cancellation law of addition does not hold. Bulletin of the American Mathematical Society. 1934; 40(12):914-20.

3. Golan JS. The theory of semirings with applications in mathematics and theoretical computer science, John Willy and Sons Inc.: New York, NY, 1992.

4. Praba B, Chandrasekaran VM, Manimaran A. Semiring on Roughsets. Indian Journal of Science and Technology. 2015 Feb; 8(3):1-7.

5. Anjaneyulu GSGN, Sanyasirao A. Distributed Group Key Management Protocol over Non-commutative Division Semirings. Indian Journal of Science and Technology. 2014 Jan; 7(6):1-6.

6. Hoorn WG, Rootoselaar B. Fundamental notions in the theory of seminear-rings. Comp. Mathematica. 1967; 18(12):65-78.

7. Weinert HJ. Seminear-rings, seminear-fields and their semigroup theoretical background. Semigroup Forum. 1982; 24(1):231-54.
8. Ihsan J. Seminear-rings characterized by their s-ideals-I. Proc. Japan Acad, Ser, 1995; 71(A): 101-03.

9. Ihsan J. Seminear-rings characterized by their $s$-ideals-II. Proc. Japan Acad, Ser, 1995; 71(A):111-13.

10. Shabir M, Ahmed I. Weakly regular seminear-rings. International Electronic Journal of Algebra. 2007; 2:114-26.

11. Zulfiqar M. A note on radicals of seminear-rings. Navi Sad J. Math. 2009; 39(1):65-8.

12. Perumal R, Balakrishnan R. Left bipotentseminear-rings. International Journal of Algebra. 2012; 6(26):1289-95.

13. Balakrishnan R, Perumal R. Left duo seminear-rings. Scientia Magna. 2012; 8(3):115-20.

14. Kazemipour SA, Fozouni M. Quotient Amenability of Banach Algebras. Indian Journal of Science and Technology. 2015 Jul; 8(13):1-7.

15. Moghaddam MA. f-best Coapproximation in Quotient Topological Vector Spaces. Indian Journal of Science and Technology. 2014 Jan; 7(12):1-4.

16. Shamik G. A characterization of ring congruences on semirings. Soochow J. Math. 1993; 19(3):305-32.

17. Howie JM. Fundamental of semigroup theory, Clarendon Press Oxford: USA, 1995.

18. Pinter C. Set theory, Addison-Wesley Publishing Co; London, 1971. 